\title{
A Joint Maximum Likelihood Approach to Frame Synchronization in Presence of Frequency Offset
}

\author{
Younghoi Koo and Yong H. Lee \\ Department of Electrical Engineering and Computer Science \\ Korea Advanced Institute of Science and Technology \\ 373-1, Kusong-dong,Yusung-gu, Taejon, 305-701, KOREA
}

\begin{abstract}
This paper proposes a new frame synchronizer that can achieve frame sync in presence of frequency offset. In particular, a maximum likelihood (ML) algorithm for joint frame synchronization, frequency and channel estimation is developed. Its derivation starts with the assumption of additive white Gaussian noise (AWGN) channels, but the results is extended to frequency selective channels. It is shown through computer simulation that the proposed scheme outperformed the existing schemes when frequency offset exists.
\end{abstract}

Index Terms - Frame synchronization, maximum likelihood, frequency offset.

\section{INTRODUCTION}

Motivated by the fact that frame synchronization is usually performed before carrier recovery is completed, frame synchronization which are tolerant of frequency and phase errors have been developed recently [1]-[4]. They are ML rules-with the exception of an ad hoc rule in [1] - for AWGN [2],[3] and frequency selective channels [4]. The ML rules have been derived following two different approaches ${ }^{1}$. One is the Bayesian approach adopted in [2],[3], which involves averaging of a probability density function (pdf) over transmitted data, frequency and phase offsets. The other is the joint estimation approach adopted in [4], which jointly achieves frame sync, frequency and channel estimation. In this case, to simplify the derivation, the rule assumes a special sync pattern which is periodically repeated.

In this paper, we first develop a new ML rule for AWGN channels following the joint estimation approach. Then we return to the problem discussed in [4], but derive an ML rule that allows an arbitrary sync pattern. The advantage of the proposed rule over the existing schemes is demonstrated through computer simulation.

\section{SIGNAL MODEL}

We consider packet transmission over a dispersive channel whose dispersion spans up to $L$ symbol intervals. It is assumed

1 Additional discussion about these approaches may be found in [5]. that a packet starts with a data sequence of length $P$ followed by a training sequence consisting of $L-1$ guard symbols and a sync pattern of duration $N_{s}$ (Fig. 1). The guard symbols are inserted so that the sync pattern is not affected by random data. After the sync pattern, data symbols are continued ${ }^{2}$. The training sequence is denoted by $\left\{s_{-L+1}, \ldots, s_{-1}, s_{0}, \ldots, s_{N_{s}-1}\right\}$ in which the first $L-1$ samples represent guard symbols. It is assumed that symbols are transmitted in $M$-ary PSK format. The received baseband signal, which is sampled with symbol interval $T_{s}$, is expressed as

$$
r_{k}=\sum_{l=0}^{L-1} h_{l} e^{j\left(\theta_{k-l}+2 \pi f_{0} T_{s} k\right)}+w_{k}
$$

where $e^{j \theta_{k}}$ is the $M$-ary phase-modulated symbol at time $\mathrm{k} ; L$ represents the channel memory; $\mathbf{h}=\left[h_{0}, h_{1}, \ldots, h_{L-1}\right]^{T}$ is a vector containing $T_{s}$-spaced samples of the channel response; and $f_{0}$ denotes the frequency offset. Complex AWGN is denoted by $w_{k}$. Its variance $\sigma_{w}^{2}=N_{0} / E_{s}$ where $E_{s}$ represents symbol energy and $N_{0}$ is the noise power. For AWGN channels, $L=1$ and $h_{0}=e^{j \phi_{0}}$ where $\phi_{0}$ is the phase offset. In this case, $r_{k}$ in (1) is reduced to

$$
r_{k}=e^{j\left(\theta_{k}+2 \pi f_{0} T_{s} k+\phi_{0}\right)}+w_{k}
$$

Throughout this paper, frame synchronization is started under the assumption that the position of packet is roughly known up to an uncertainty of $\mu \in\{0,1, \ldots, P+L-1\}$ symbols (such coarse frame sync can be achieved by automatic gain control [4]). The test metric for frame synchronization is evaluated from $N$ received samples $\left\{r_{0}, r_{1}, \ldots, r_{N-1}\right\}$. The observation window size $N \geq N_{s}+P+L-1$ because otherwise, some received samples corresponding to the sync pattern may be excluded.

\footnotetext{
2 The training sequence in this packet is a midamble, and thus the proposed synchronizer which will be derived based on this packet is most suitable for systems such as the GSM [6] that employs a midamble. To consider a preamble some minor modification is necessary.
} 


\section{Derivation of the Proposed Frame SYNCHRONIZATION}

\section{A. Frame Synchronization in AWGN channel}

Fig. 2 illustrates the received sequence $\mathbf{r}=$ $\left\{r_{0}, r_{1}, \ldots, r_{N-1}\right\}$ in the observation window and the corresponding transmitted sequence for an AWGN channel. If the sync pattern starts at the $\mu$-th position, $\mu \in[0, P]$, then the conditional pdf of $\mathbf{r}$ assuming $\mu, f_{0}, \phi_{0}$ and $\mathbf{d}$ is given by

$$
p\left(\mathbf{r} \mid \mu, f_{0}, \phi_{0}, \mathbf{d}\right)=\prod_{k=0}^{N-1} \frac{E_{s}}{\pi N_{0}} e^{-\left|r_{k}-e^{j\left(\theta_{k}+2 \pi f_{0} T_{s} k+\phi_{0}\right)}\right|^{2} \frac{E_{s}}{N_{0}}} .
$$

The joint ML estimates of $\mu, f_{0}$ and $\phi_{0}$ are obtained by maximizing $p\left(\mathbf{r} \mid \mu, f_{0}, \phi_{0}\right)$ with respect to $\mu, f_{0}$ and $\phi_{0}$. Evaluating the average of (3) over all possible data $\{\mathbf{d}\}$, we get

$$
p\left(\mathbf{r} \mid \mu, f_{0}, \phi_{0}\right)=\frac{1}{M^{N-N_{s}}} \sum_{\text {all } \mathbf{d}} p\left(\mathbf{r} \mid \mu, f_{0}, \phi_{0}, \mathbf{d}\right) .
$$

To simplify this expression, the information symbol $e^{j \theta_{k}}$ is approximated as $e^{j \alpha}$ where $\alpha$ has a uniform distribution over $(-\pi, \pi)[1, \mathrm{p} .283]$. Then

$$
\begin{aligned}
p(\mathbf{r} \mid & \left.\mu, f_{0}, \phi_{0}\right) \\
\cong & \left(\frac{E_{s}}{\pi N_{0}}\right)^{N} \prod_{k=\mu}^{\mu+N_{s}-1} e^{-\left|r_{k}-s_{k-\mu} e^{j\left(2 \pi f_{0} T_{s} k+\phi_{0}\right)}\right|^{2} \frac{E_{s}}{N_{0}}} \\
& \cdot \prod_{k \notin \Omega} \frac{1}{2 \pi} \int_{-\pi}^{\pi} e^{-\left|r_{k}-e^{j\left(\alpha+2 \pi f_{0} T_{s} k+\phi_{0}\right)}\right|^{2} \frac{E_{s}}{N_{0}}} d \alpha \\
= & \left(\frac{E_{s}}{\pi N_{0}}\right)^{N} \prod_{k=0}^{N-1} e^{-\left(\left|r_{k}\right|^{2}+1\right) E_{s} / N_{0}} \\
& \cdot \prod_{k=\mu}^{\mu+N_{s}-1} e^{\frac{2 E_{s}}{N_{0}} \operatorname{Re}\left\{r_{k} s_{k-\mu}^{*} e^{-j\left(2 \pi f_{0} T_{s} k+\phi_{0}\right)}\right\}} \\
& \cdot \prod_{k \notin \Omega} I_{0}\left(\frac{2 E_{s}}{N_{0}}\left|r_{k} e^{-j\left(2 \pi f_{0} T_{s} k+\phi_{0}\right)}\right|\right)
\end{aligned}
$$

where $\boldsymbol{\Omega}=\left\{\mu, \mu+1, \ldots, \mu+N_{s}-1\right\}$ and $I_{0}(x)=$ $\frac{1}{2 \pi} \int_{-\pi}^{\pi} e^{x \cos \theta} d \theta$ is the zeroth-order modified Bessel function of first kind. The conditional pdf in (5) is further simplified by approximating $I_{0}(x)$ as $e^{|x|} / \sqrt{2 \pi}$ [7]:

$$
\begin{aligned}
& p\left(\mathbf{r} \mid \mu, f_{0}, \phi_{0}\right) \cong C(\mathbf{r}) \\
& \cdot \prod_{k=\mu}^{\mu+N_{s}-1} e^{\frac{2 E_{s}}{N_{0}} \operatorname{Re}\left\{r_{k} s_{k-\mu}^{*} e^{-j\left(2 \pi f_{0} T_{s} k+\phi_{0}\right)}\right\}} \cdot \prod_{k \notin \Omega} e^{\left(\frac{2 E_{s}}{N_{0}}\left|r_{k}\right|\right)}
\end{aligned}
$$

where $C(\mathbf{r})=\left(\frac{E_{s}}{\pi N_{0}}\right)^{N} \prod_{k=0}^{N-1} e^{-\left(\left|r_{k}\right|^{2}+1\right) E_{s} / N_{0}} \frac{1}{(\sqrt{2 \pi})^{N-L}}$. After taking the logarithm, dropping the terms independent of $\mu, f_{0}$ and $\phi_{0}$ and subtracting a constant $\sum_{k=0}^{k=N-1}\left|r_{k}\right|$, the following test function is obtained:

$$
\begin{aligned}
& \Lambda\left(\mu, f_{0}, \phi_{0}\right) \\
& \triangleq \sum_{k=\mu}^{\mu+N_{s}-1} \operatorname{Re}\left\{r_{k} s_{k-\mu}^{*} e^{-j\left(2 \pi f_{0} T_{s} k+\phi_{0}\right)}\right\}-\sum_{k=\mu}^{\mu+N_{s}-1}\left|r_{k}\right| \\
& \quad=\sum_{l=0}^{N_{s}-1} \operatorname{Re}\left\{r_{l+\mu} s_{l}^{*} e^{-j\left(2 \pi f_{0} T_{s} l+\phi_{0}^{\prime}\right)}\right\}-\sum_{l=0}^{N_{s}-1}\left|r_{l+\mu}\right| \\
& \triangleq \Lambda\left(\mu, f_{0}, \phi_{0}^{\prime}\right)
\end{aligned}
$$

where $l=k-\mu$ and $\phi_{0}^{\prime}=\phi_{0}+2 \pi f_{0} T_{s} \mu$. When the frequency and phase offsets are zero, this expression reduces to the ML rule for high SNR derived in [8]. Maximization of $\Lambda\left(\mu, f_{0}, \phi_{0}^{\prime}\right)$ with respect to $\mu, f_{0}$, and $\phi_{0}^{\prime}$ can be achieved through the following three step procedure $[1, \text { p. 248] }]^{3}$ : First maximize $\Lambda\left(\mu, f_{0}, \phi_{0}^{\prime}\right)$ with respect to $\phi_{0}^{\prime}$ for each possible $\mu$ and $f_{0}$, Specifically, an estimate of $\phi_{0}^{\prime}$ is obtained as a function of $\mu$ and $f_{0}$ :

$$
\hat{\phi}_{0}^{\prime}\left(\mu, f_{0}\right)=\arg \max _{\phi_{0}^{\prime}} \Lambda\left(\mu, f_{0}, \phi_{0}^{\prime}\right) .
$$

Second derive an estimate of $f_{0}$ as a function of $\mu$, which is expressed as

$$
\hat{f}_{0}(\mu)=\arg \max _{f_{0}} \Lambda\left(\mu, f_{0}, \phi_{0}^{\prime}=\hat{\phi}_{0}^{\prime}\left(\mu, f_{0}\right)\right)
$$

Third select $\mu$ with the largest likelihood

$$
\hat{\mu}=\arg \max _{\mu} \Lambda\left(\mu, f_{0}=\hat{f}_{0}(\mu), \phi_{0}^{\prime}=\hat{\phi}_{0}^{\prime}\left(\mu, \hat{f}_{0}(\mu)\right)\right) .
$$

To derive (8), $\Lambda\left(\mu, f_{0}, \phi_{0}^{\prime}\right)$ is differentiated with respect to $\phi_{0}^{\prime}$ and the result is set to zero. This yields

$$
\hat{\phi}_{0}^{\prime}\left(\mu, f_{0}\right)=\arg \left\{\sum_{l=0}^{N_{s}-1} r_{l+\mu} s_{l}^{*} e^{-j 2 \pi f_{0} T_{s} l}\right\}
$$

Using (11) in (7), we get

$$
\Lambda\left(\mu, f_{0}, \phi_{0}^{\prime}=\hat{\phi}_{0}^{\prime}\left(\mu, f_{0}\right)\right) \triangleq \Lambda^{\prime}\left(\mu, f_{0}\right)-\sum_{l=0}^{N_{s}-1}\left|r_{l+\mu}\right|
$$

where

$$
\Lambda^{\prime}\left(\mu, f_{0}\right)=\left|\sum_{l=0}^{N_{s}-1} r_{l+\mu} s_{l}^{*} e^{-j 2 \pi f_{0} T_{s} l}\right|
$$

Now $\hat{f}_{0}(\mu)$ in (9) can be obtained by maximizing $\Lambda^{\prime}\left(\mu, f_{0}\right)$. Differentiating the square of $\hat{f}_{0}(\mu)$ with respect to $f_{0}$ and setting the result equal to zero yields

$$
\sum_{l=1}^{N_{s}-1} \sum_{m=1}^{N_{s}-1}(l-m) r_{l+\mu} s_{l}^{*} r_{m+\mu}^{*} s_{m} e^{-j 2 \pi f_{0} T_{s}(l-m)}=0 .
$$

\footnotetext{
${ }^{3}$ Since $\mu, f_{0}$ and $\phi_{0}^{\prime}$ are finite, $\Lambda\left(\mu, f_{0}, \phi_{0}^{\prime}\right)$ can also be maximized through an exhaustive search [5].
} 
or equivalently,

$$
\begin{aligned}
& \operatorname{Im}\left\{\sum_{l=1}^{N_{s}-1} l \rho(l) e^{-j 2 \pi f_{0} T_{s} l}\right\} \\
& =\sum_{l=1}^{N_{s}-1} l|\rho(l)| \cdot \sin \left(\arg [\rho(l)]-j 2 \pi f_{0} T_{s} l\right)=0
\end{aligned}
$$

where

$$
\rho(l)=\sum_{m=l}^{N_{S}-1} r_{m+\mu} s_{m}^{*} r_{m-l+\mu}^{*} s_{m-l}
$$

Note that $\arg [\rho(l)] \simeq 2 \pi f_{0} T_{s} l$ (in fact, $\arg [\rho(l)]=$ $2 \pi f_{0} T_{s} l$ for the noise-free case). Therefore, $\sin (\arg [\rho(l)]-$ $\left.2 \pi f_{0} T_{s} l\right) \simeq \arg [\rho(l)]-2 \pi f_{0} T_{s} l$ and the following estimate is obtained from (15):

$$
\hat{f}_{0}(\mu)=\frac{1}{2 \pi T_{s}} \sum_{l=1}^{R} w_{l} \arg \{\rho(l)\}
$$

where $1 \leq R \leq N_{s}-1$ and

$$
w_{l}=\frac{l|\rho(l)|}{\sum_{m=1}^{R} m^{2}|\rho(m)|}
$$

It should be pointed out that direct computation of (15) leads to $\hat{f}_{0}(\mu)$ with $R=N_{s}-1$. The design parameter $R$ enables us to adjust the acquisition range of the frequency estimate, which is given by

$$
\left|\hat{f}_{0}(\mu)\right|<\frac{1}{2 R T_{s}}
$$

(see Eq. (13)). The proposed frame synchronization rule is obtained from (10) and (12):

$$
\hat{\mu}=\arg \max _{\mu}\left\{\left|\sum_{l=0}^{N_{s}-1} r_{l+\mu} s_{l}^{*} e^{-j 2 \pi \hat{f}_{0}(\mu) T_{s} l}\right|-\sum_{l=0}^{N_{s}-1}\left|r_{l+\mu}\right|\right\} .
$$

where $\hat{f}_{0}(\mu)$ is given by (17).

\section{B. Extension to frequency selective channels}

For frequency selective channels, to simplify the derivation, we employ a subwindow of length $N_{s}$ observing $\mathbf{r}_{\mu}=$ $\left[r_{\mu}, r_{\mu+1}, \ldots, r_{\mu+N_{s}-1}\right]^{T}$ for each $\mu \in\{0,1, \ldots, P+L-1\}$ and derive the conditional pdf of $\mathbf{r}_{\mu}$. Under the assumption that $\mu$ is the correct sync pattern start position, $\mathbf{r}_{\mu}$ only corresponds to the training sequence(Fig. 3); and thus its conditional pdf does not dependent upon random data within the packet. This fact greatly simplifies the derivation, because averaging the conditional pdf over all possible data sequence becomes unnecessary.
The conditional pdf of $\mathbf{r}_{\mu}$ assuming $\mu, f_{0}$ and $\mathbf{h}$ takes the form

$$
\begin{aligned}
& p\left(\mathbf{r}_{\mu} \mid \mu, f_{0}, \mathbf{h}\right)=\left(\frac{E_{s}}{\pi N_{0}}\right)^{N_{s}} \\
& \cdot \exp \left\{-\left(\mathbf{r}_{\mu}-\boldsymbol{\Gamma}\left(f_{0}\right) \mathbf{A h}\right)^{H}\left(\mathbf{r}_{\mu}-\boldsymbol{\Gamma}\left(f_{0}\right) \mathbf{A h}\right) \frac{E_{s}}{N_{0}}\right\}
\end{aligned}
$$

where $\boldsymbol{\Gamma}\left(f_{0}\right)$ is a diagonal matrix

$$
\boldsymbol{\Gamma}\left(f_{0}\right)=\operatorname{diag}\left\{1, e^{j 2 \pi f_{0}}, e^{j 4 \pi f_{0}}, \ldots, e^{j 2 \pi(L-1) f_{0}}\right\}
$$

and $\mathbf{A}$ is a $N_{s} \times L$ matrix with entries

$$
[\mathbf{A}]_{i, j}=s_{i-j} 0 \leq i \leq N_{s}-1,0 \leq j \leq L-1 .
$$

After taking the logarithm and dropping the terms independent of $\mu, f_{0}$ and $\mathbf{h}$, we get

$$
\Lambda\left(\mu, f_{0}, \mathbf{h}\right)=-\left(\mathbf{r}_{\mu}-\boldsymbol{\Gamma}\left(f_{0}\right) \mathbf{A h}\right)^{H}\left(\mathbf{r}_{\mu}-\boldsymbol{\Gamma}\left(f_{0}\right) \mathbf{A h}\right) .
$$

To maximize $\Lambda\left(\mu, f_{0}, \mathbf{h}\right)$ with respect to $\mu, f_{0}$ and $\mathbf{h}$, the three step approach introduced in the previous section is applied. First a channel estimate is found by setting the derivative of (24) equal to zero. The result is:

$$
\hat{\mathbf{h}}\left(\mu, f_{0}\right)=\left(\mathbf{A}^{H} \mathbf{A}\right)^{-1} \mathbf{A}^{H} \boldsymbol{\Gamma}\left(f_{0}\right)^{H} \mathbf{r}_{\mu} .
$$

Substituting $\hat{\mathbf{h}}\left(\mu, f_{0}\right)$ in (25) for $\mathbf{h}$ in (24),

$$
\begin{aligned}
& \Lambda\left(\mu, f_{0}, \mathbf{h}=\hat{\mathbf{h}}\left(\mu, f_{0}\right)\right)=\mathbf{r}_{\mu}{ }^{H} \boldsymbol{\Gamma}\left(f_{0}\right) \mathbf{B} \boldsymbol{\Gamma}\left(f_{0}\right)^{H} \mathbf{r}_{\mu}-\mathbf{r}_{\mu}{ }^{H} \mathbf{r}_{\mu} \\
& =2 \operatorname{Re}\left\{\sum_{l=0}^{N_{s}-1} \rho(l) e^{-j 2 \pi f_{0} T_{s} l}\right\}-\rho(0)-\sum_{l=0}^{N_{s}-1}\left|r_{l+\mu}\right|^{2}
\end{aligned}
$$

where $\mathbf{B}=\mathbf{A}\left(\mathbf{A}^{H} \mathbf{A}\right)^{-1} \mathbf{A}^{H}$,

$$
\rho(l)=\sum_{m=l}^{N_{S}-1}[\mathbf{B}]_{l-m, m} r_{m+\mu} r_{m-l+\mu}^{*},
$$

and $[\mathbf{B}]_{i, j}$ is the $(i, j)$-entry of $\mathbf{B}$. For each $\mu, \hat{f}_{0}(\mu)$ is estimated by

$$
\hat{f}_{0}(\mu)=\arg \max _{f_{0}}\left[2 \operatorname{Re}\left\{\sum_{l=0}^{N_{s}-1} \rho(l) e^{-j 2 \pi f_{0} T_{s} l}\right\}-\rho(0)\right] .
$$

This estimate can be efficiently computed using fast Fourier transform (FFT)[5]. Using (27) in (26), then the proposed frame sync rule for frequency selective channels is obtained:

$$
\hat{\mu}=\arg \max _{\mu}\left\{\mathbf{r}_{\mu}{ }^{H} \boldsymbol{\Gamma}\left(\hat{f}_{0}(\mu)\right) \mathbf{B} \boldsymbol{\Gamma}\left(\hat{f}_{0}(\mu)\right)^{H} \mathbf{r}_{\mu}-\mathbf{r}_{\mu}{ }^{H} \mathbf{r}_{\mu}\right\} .
$$




\section{Simulation Results}

False acquisition probabilities (FAPs)of the proposed rules were investigated by simulation with the following parameters: $M=4$ (QPSK), $N=80$ and $P=20$. The sync pattern of length $N_{s}=16$ was taken from the midamble of the GSM, which is given by

$$
\{1,-j, 1, j, 1,-j,-1,-j,-1, j,-1,-j,-1, j,-1,-j\} .
$$

The frequency selective channel was modeled as:

$$
h(t)=\sum_{l=0}^{L-1} h_{l} \delta\left(t-l T_{s}\right)
$$

where $\left\{h_{l}\right\}$ are independently identically distributed complex Gaussian random variables with zero mean and variance $1 / L$. Simulations use 500,000 independent frames and the FAP was empirically estimated by counting the number of frame synchronization failures. For comparison, we also considered the conventional correlation rule and the rule in [3] for AWGN channels and the algorithm in [4] for dispersive channels. The rule in [3] is expressed as

$$
\begin{aligned}
\hat{\mu} & =\arg \max _{\mu} \sum_{i=1}^{N_{s}-1}\left\{\left|\sum_{k=i}^{N_{s}-1} r_{\mu+k}^{*} s_{k} * r_{\mu+k-i} s_{k-i}^{*}\right|\right. \\
& \left.-\sum_{\mu+i}^{\mu+N_{s}-1}\left|r_{k}\right|\left|r_{k-i}\right|\right\} .
\end{aligned}
$$

The rule in [4] is computed by iteratively minimizing the likelihood function $\Lambda\left(\mu, f_{0}\right)$ starting with initial frequency estimate $\hat{f}_{0}^{(1)}=\arg [\rho(L)]$ where $\rho(l)$ is given by (16). The likelihood function $\Lambda\left(\mu, f_{0}\right)$ is given by

$$
\begin{aligned}
& \Lambda\left(\mu, f_{0}\right)= \\
& \quad-\rho(0)-2 \sum_{l=1}^{N_{s}-1} \frac{|\rho(l)|}{\rho(0)} \cos \left(2 \pi l f_{0} T_{s}-\arg [\rho(l)]\right)
\end{aligned}
$$

This rule assumes the repeated CAZAC (constant-amplitude, zero autocorrelation) sequence[1, p.788]. Therefore, the CAZAC sequence was employed in implementing the rule in (33), while all the other rules in the simulation used the training sequence in (29). The robustness of the frame synchronizers was examined by estimating the FAPs for various normalized frequency offsets $\left(f_{0} T_{s}\right)$ in between 0 and 0.3 , while fixing $E_{b} / N_{0}$ at $3 \mathrm{~dB}$. The results are shown in Fig. 4. As expected, the correlation rule performed worst. The FAP of the proposed rule decreased as $\mathrm{R}$ increased, at the expense of narrower frequency acquisition range (see (19)). The rule in [3] was most robust to frequency offsets, but its FAPs were larger than those of the proposed with $R=4$ and 10 .

Fig. 5 and 6 compare performances of the rules when $f_{0} T_{s}$ was uniformly distributed over $\left[-f_{m}, f_{m}\right]$, where $f_{m}=0.01$ and 0.1 for Fig. 5 and Fig. 6, respectively. Again, the proposed rule outperformed the existing rules.

Fig. 7 shows the results for the frequency selective channel. The proposed rule performed better than the rule in [4]. This happened because of the periodically repeated CAZAC sequence: due to the repetition, the test function in (33) tends to generate periodically repeated peaks as $\mu$ varies from 0 to $P+L-1$, which degraded the performance of frame synchronization.

\section{CONCLUSION}

ML-type frame synchronizers that jointly achieve frame sync, frequency and channel estimation have been derived and advantages of the proposed techniques over the existing ones have been demonstrated through computer simulation. Futher work in this direction will be concentrated on extending the proposed schemes to the case of transmitter and receiver diversity provided that the frequency offset is the same for each diveristy branch.

\section{REFERENCES}

[1] H. Meyr, M. Moeneclaey and S. A. Fechtel Ditital Communication Receivers., New York: John Wiley \& Sons, 1998.

[2] J. M. Gansman, and M. P. Fitz and J. V. Krogmeier, "Optimum and suboptimum frame synchronization for pilot-symbol-assisted modulation," IEEE Trans. Commun., vol. 45, pp. 1327-1337, Oct. 1997.

[3] Z. Y. Choi and Y. H. Lee, "On the use of double correlation for frame synchronization in the presence of frequency offset," IEEE Trans. Commun., Accepted for publication.

[4] S. A. Fechtel and H. Meyr, "Fast frame synchronization, frequency offset estimation and channel acqusition for spontaneous transmission over unknown frequency-selective radio channels," Proc. IEEE PIMRC'93, pp. 229-233.

[5] M. Morelli and U. Mengali, "Carrier-frequency estimation for transmissions over selective channels," IEEE Trans. Commun., vol. 48, pp. 15801589, Sept. 2000.

[6] Radio Transmission and Reception, GSM Recommendation 0505, ETSI/PT 12, V. 3.11.0, Jan. 1990.

[7] G. T. Lui and H. H. Tan, "Frame synchronization for Gaussian channels," IEEE Trans. Commun., vol. 35, pp. 818-829, Aug. 1987.

[8] P. T. Nielsen, "Some optimum and suboptimum frame synchronization for binary data in Gaussian noise," IEEE Trans. Commun., vol. 21, pp. 770-772, June. 1973. 


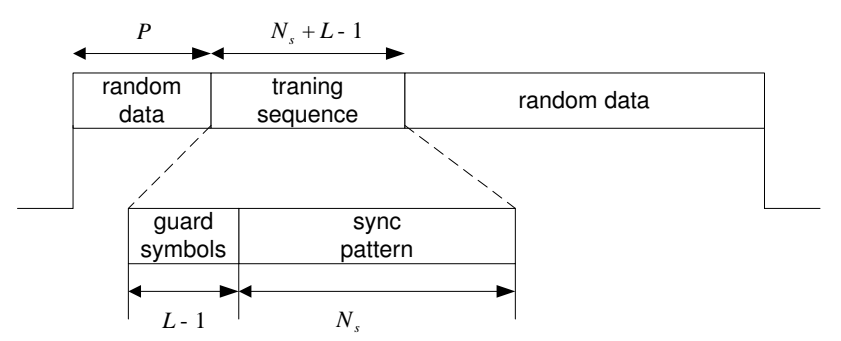

Fig. 1. Frame Structure.

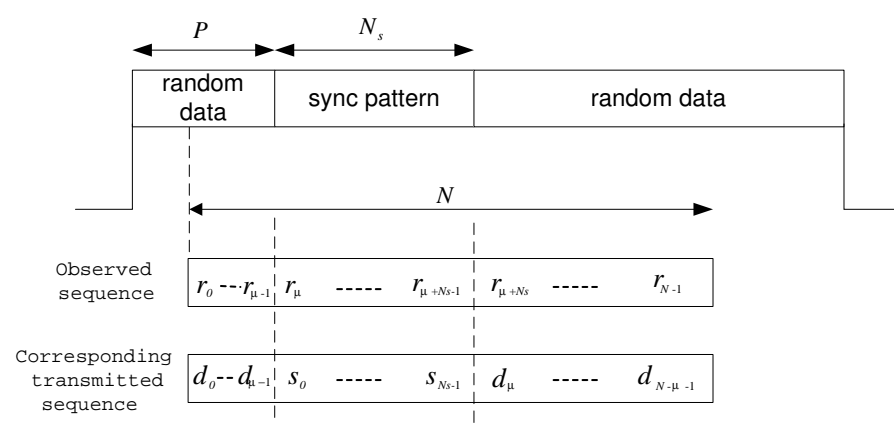

Fig. 2. Observed sequence and corresponding transmitted data in an AWGN channel, where $0 \leq \mu \leq P$.

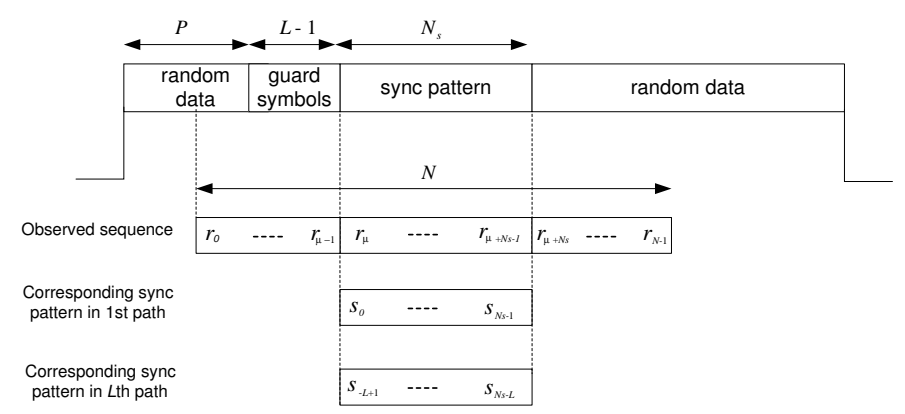

Fig. 3. Observed sequence and corresponding transmitted data in a frequency selective channel, where $0 \leq \mu \leq P+L-1$

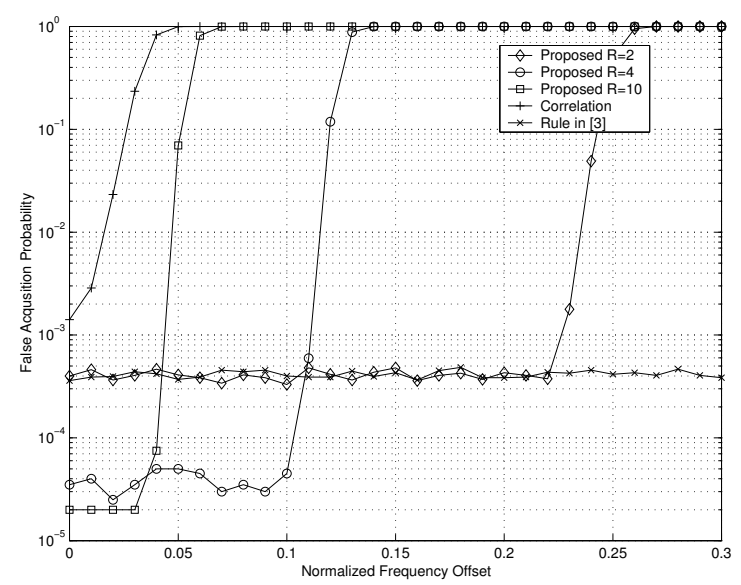

Fig. 4. False acquisition probability vs. normalized frequency offset when $E_{b} / N_{o}$ is $3 \mathrm{~dB}$.

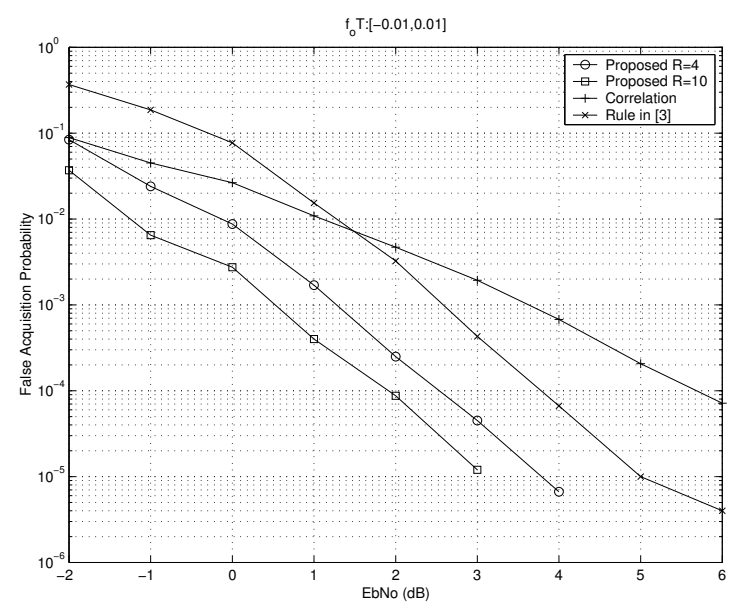

Fig. 5. False acquisition probability vs. signal to noise ratio.

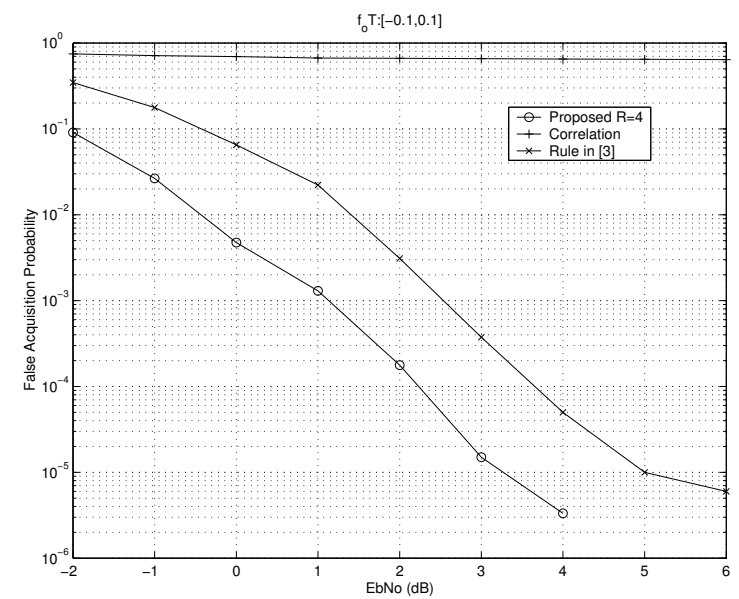

Fig. 6. False acquisition probability vs. signal to noise ratio.

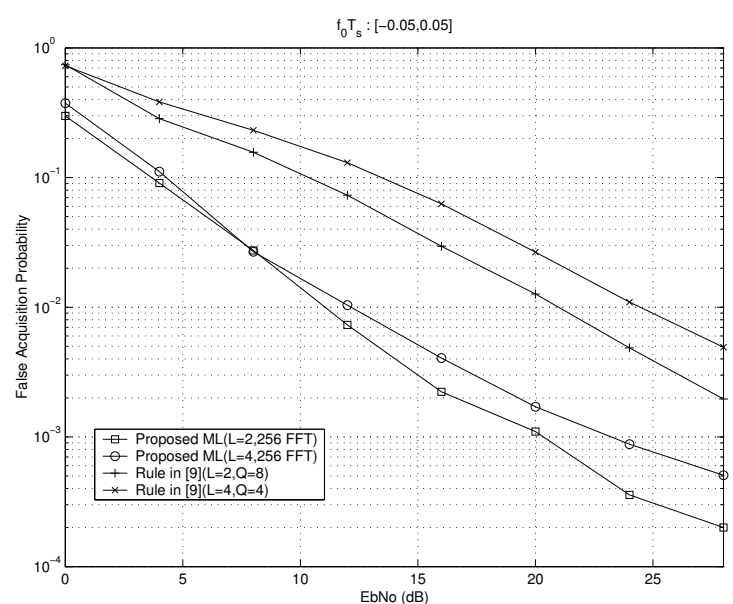

Fig. 7. Probability of false acquisition for the frequency selective fading channel. The normalized frequency offset was generated randomly over $[-0.05,0.05]$. $L$ is the channel length. 